\title{
Nodal Stub-Release in All-Optical Networks
}

\author{
Ruepp, Sarah Renée; Buron, Jakob Due; Andriolli, Nicola; Dittmann, Lars
}

Published in:

IEEE Communications Letters

Link to article, DOI:

10.1109/LCOMM.2008.071560

Publication date:

2008

Document Version

Publisher's PDF, also known as Version of record

Link back to DTU Orbit

\section{Citation (APA):}

Ruepp, S. R., Buron, J. D., Andriolli, N., \& Dittmann, L. (2008). Nodal Stub-Release in All-Optical Networks. IEEE Communications Letters, 12(1), 47-49. https://doi.org/10.1109/LCOMM.2008.071560

\section{General rights}

Copyright and moral rights for the publications made accessible in the public portal are retained by the authors and/or other copyright owners and it is a condition of accessing publications that users recognise and abide by the legal requirements associated with these rights.

- Users may download and print one copy of any publication from the public portal for the purpose of private study or research.

- You may not further distribute the material or use it for any profit-making activity or commercial gain

- You may freely distribute the URL identifying the publication in the public portal 


\title{
Nodal Stub-Release in All-Optical Networks
}

\author{
Sarah Ruepp, Student Member, IEEE, Jakob Buron, Student Member, IEEE, Nicola Andriolli, \\ and Lars Dittmann, Member, IEEE
}

\begin{abstract}
In wavelength-routed networks, the scarcity of wavelength-converters severely impacts successful connection recovery. This can be mitigated by releasing the connection's stubs (i.e. the surviving upstream and downstream span and node resources) prior to the restoration process. Releasing span resources however heavily complicates the reversion process.

In this study, we present a nodal stub-release method, where only node resources (i.e. wavelength-converters) are released, while the stubs' span resources are kept occupied. Our simulation results show that the nodal stub-release method can match the performance of full stub-release (releasing both span and node resources) while keeping complexity low.
\end{abstract}

Index Terms-Restoration, stub-release, wavelengthconversion.

\section{INTRODUCTION}

$\mathbf{I}$ $\mathrm{N}$ wavelength-routed networks, the traffic is switched at wavelength granularity. Emerging technologies allow for all-optical conversion between different wavelengths, which gives greater flexibility when setting up lightpaths [1]. These components are however costly and hence only sparingly provided and possibly shared [2]. Two different resources need to be considered when setting up a lightpath: the span resources (i.e. the wavelength channels) and the node resources (i.e. wavelength-converters). The configuration of wavelength channels requires signalling between the end nodes in a dynamic network environment employing two-way signalling. In contrast, wavelength-converters (WCs) are managed locally in each node and can easily be reassigned.

Optical networks can be affected by natural and manmade failures, and the traffic flows must thereafter be restored via routes that avoid the faulty spans or nodes. In network restoration, the affected lightpaths are restored after the failure occurs on the free capacity throughout the network. This approach has the benefit of higher flexibility and robustness towards a variety of failure scenarios [3], but it also means that many lightpaths require access to limited resources during a short timeframe. Especially the wavelength-converters play an important role in the restoration process, as their exhaustion severely diminishes the chance of successful recovery [4], [5].

When lightpaths are affected by a failure, a decision regarding the still functioning segments of the lightpath (called the stubs) has to be made before the restoration process can

Manuscript received September 21, 2007. The associate editor coordinating the review of this letter and approving it for publication was N. Ghani. This work has been partially supported by the European Commission through the Network of Excellence e-Photon/ONe+.

S. Ruepp, J. Buron, and L. Dittmann are with COM-DTU, Technical University of Denmark, Kgs. Lyngby, Denmark (e-mail: \{sr, jbu, ld\}@ com.dtu.dk).

N. Andriolli is with Scuola Superiore Sant'Anna, Pisa, Italy (e-mail: nick@sssup.it).

Digital Object Identifier 10.1109/LCOMM.2008.071560. be initiated. Either, the stubs are kept occupied, meaning that the affected lightpaths have to be restored using remaining network resources. Alternatively, the stubs can be released, freeing an often considerable amount of network resources which can be used as spare capacity in the restoration process.

Once the failure is repaired, the restored lightpaths can either continue operating on their backup paths, or the backup paths can be torn down and the traffic is re-accommodated on its original paths. The latter approach is called reversion.

The complexity of the reversion operation is highly dependent on the use of stub-release. If the stubs were not released, the pre-failure path would be in operation as soon as the failure is fixed, and the backup path could be torn down. If stubrelease was used, the pre-failure path must be re-provisioned and re-signalled to achieve reversion, even if the connection details have been saved before its release. This increases both the reversion time and the complexity.

Previous studies [3], [6] considered the effect of releasing stub capacity, showing that stub-release can increase the capacity efficiency; the actual benefit is however dependent on the studied network scenario. In these studies, only span resources are considered during the stub-release process, and they do not address the challenge of making highly scarce and needed nodal resources, such as WCs, widely available for the restoration process. The performance of full stub-release (releasing span and node resources) was investigated in [5].

In this paper, we propose a nodal stub-release method where only nodal resources (i.e. WCs) are released during the stubrelease operation, whereas the span resources of the stubs are kept occupied. This makes the scarce WC resources available in the recovery phase and allows fast and simple reversion to the pre-failure network configuration. By simulation, we validate the approach in a dynamic restoration study in three networks with sparse WC availability.

\section{Nodal Stub-Release Method}

Due to the high price of WCs, it is a realistic assumption that only a limited number of these components is provided in the network nodes. As shown in [5], WCs are often the bottleneck for connection restoration. Hence, we propose to solely release the WCs along the stub path, while keeping the span resources (i.e. the wavelengths) occupied.

The nodal stub-release method has the advantage of providing access to highly demanded WCs during the recovery phase. At the same time, the reversion process is kept simple as the re-negotiation of span resources is avoided.

\section{A. Restoration and Reversion Signalling}

The concept of nodal stub-release and reversion is shown in Fig. 1. To perform nodal stub-release, a notification message 
(a)

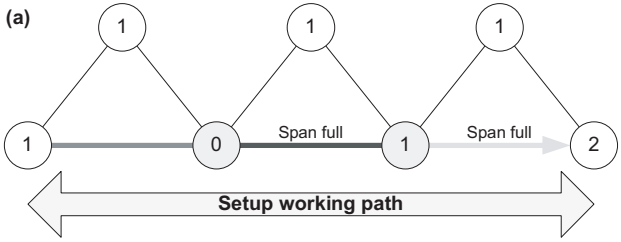

(b)
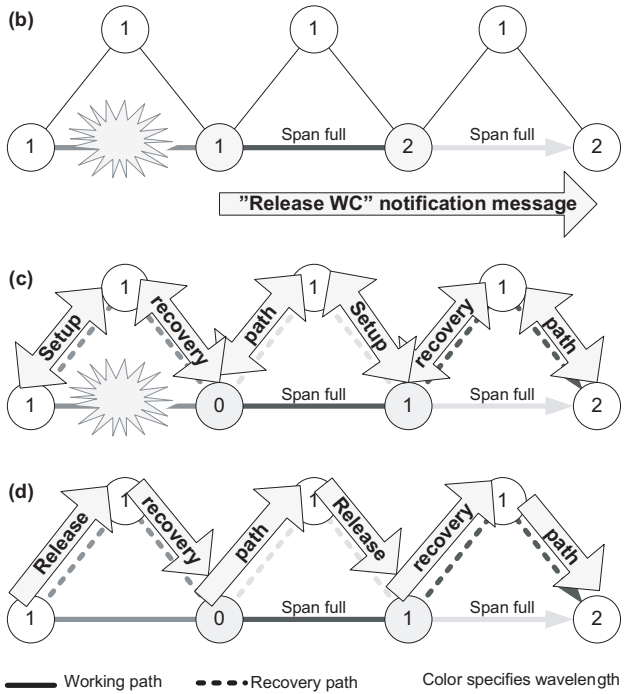

Fig. 1. Recovery and reversion of one lightpath with nodal stub-release Numbers in nodes indicate available WCs. (a) failure-free lightpath, (b) nodal stub-release (WC release), (c) restored lightpath, (d) reversion process.

is propagated from the failure-adjacent node (towards the destination node, and possibly source node, depending on the used restoration method), which instructs the nodes along the route to release the WCs of the failure affected lightpaths, while the span resources remain untouched. The released WCs can then be used by restoration requests to restore lightpaths. Once the failed span is repaired, the traffic is reverted to its original paths. While full stub-release requires re-provisioning and re-signaling of the working path and teardown of the recovery path, nodal stub-release only entails tear-down of the recovery path. Since the span resources have not been released and the originally used WCs become available by releasing the recovery path, the working path can continue its operation without requiring re-signaling. Nodal stub-release hence reduces the control plane load and increases the reversion speed.

\section{Simulation STUdy}

In this study, we investigate how the nodal stub-release method performs in terms of achieved restoration percentage. The following stub-release methods are compared:

1) No stub-release: The stubs' span and node resources (i.e. WCs) are kept occupied during restoration.

2) Full stub-release: Both the stubs' span resources and the corresponding WCs are released.

3) Nodal stub-release: Only the stubs' WCs are released, the span resources are kept occupied.

We are using Generalized Multi-Protocol Label Switching (GMPLS) control plane signaling with standard protocol extensions (i.e. the Label Set) [7]. As restoration method, we are using local-to-egress restoration [5], which combines the advantages of the well-known span and path restoration methods, such as short notification time and high resource efficiency. In local-to-egress restoration, failure affected connections are restored between the upstream failure-adjacent node and the destination node. We assume a node architecture where the WCs are shared in a per-node converter bank [1].

We evaluate the recovery performance in the NSFNET, the Pan-European triangular topology network and a German network, using OPNET Modeler [8]. The network properties are shown in Table I.

TABLE I

NETWORK SCENARIOS

\begin{tabular}{|c|c|c|c|}
\hline Network & Nodes & Spans & Nodal degree \\
\hline German & 11 & 33 & 6.00 \\
NSFNET & 14 & 22 & 3.14 \\
Pan-EU & 28 & 61 & 4.36 \\
\hline
\end{tabular}

Each span is bidirectional and has a capacity of 10 wavelengths. The network is occupied with unidirectional lightpaths, each requiring a full wavelength channel, based on a uniform distribution of the source/destination pairs. Established lightpaths are not torn down and the setup operation continues until a specified average network load is reached. When the desired load is reached, a span is failed and either no, nodal or full stub-release is executed before recovery is attempted. If a recovery request experiences blocking, the span that causes the blocking is excluded from the route computation process and restoration is reattempted after a backoff period. When all lightpaths are either restored or found unrecoverable, the network is reverted to its pre-failure state before the next span failure is evaluated. This procedure continues until the failure of all spans in the network has been simulated. This procedure is repeated 20 times with different random seeds. The results of the 20 repetitions are averaged and confidence intervals at $95 \%$ confidence level are calculated.

\section{RESUlts}

In Fig. 2 (a)-(c), the recovery percentage is illustrated for a varying number of WCs per node, while the average network load is kept constant at 0.5 . These results focus on the performance of nodal stub-release relative to full and no stubrelease, rather than the absolute recovery percentages, which are dependent on external factors such as span dimensioning and WC distribution. For all three networks, if 0 WCs are available in the nodes, the nodal stub-release scheme and the no stub-release scheme have the same performance, which is expected. Only the full stub-release scheme, where span resources are released, increases the restoration percentage.

In (a) German network (i.e. the densest tested topology), the highest recovery percentages are achieved. The nodal stubrelease scheme equals the full stub-release scheme, while the no stub-release scheme achieves lower performance. If more than 10 WCs are available per node, all three schemes achieve the same performance, since the abundance of WCs and multiple recovery path options due to a high nodal degree 


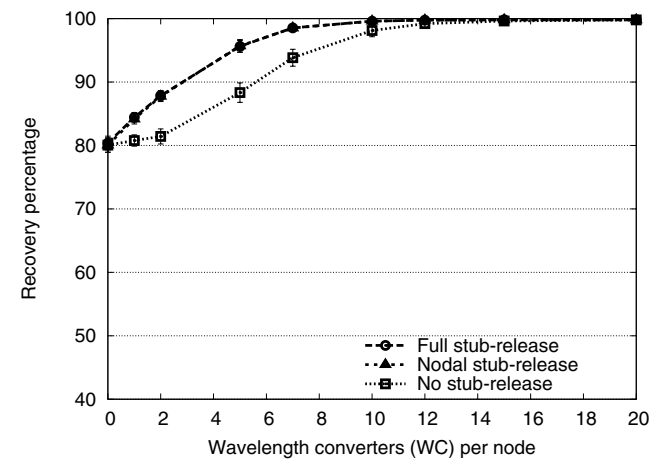

(a) German network with varying number of WCs

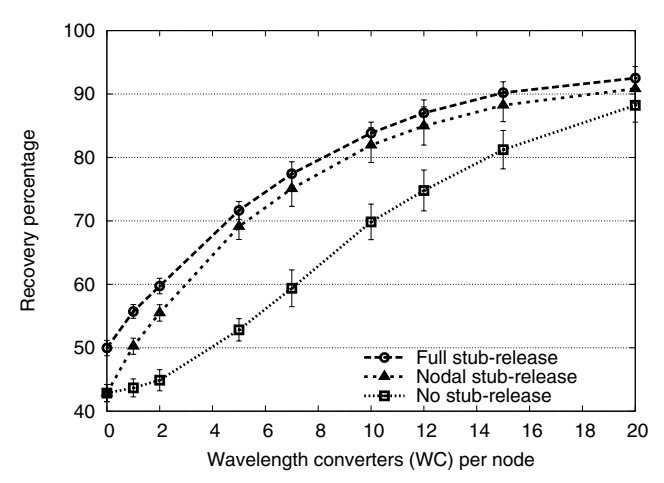

(c) Pan-European network with varying number of WCs

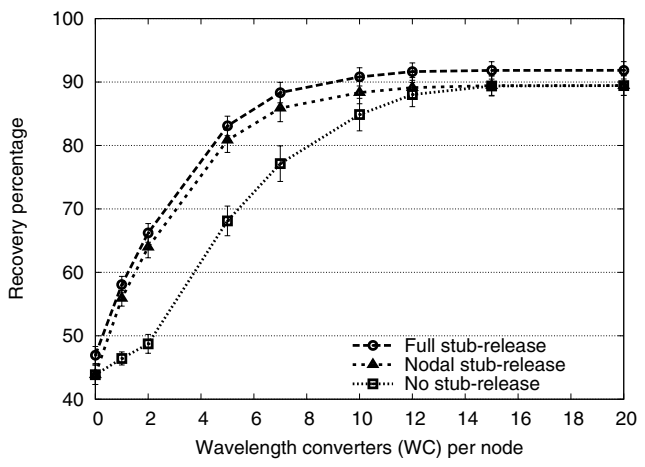

(b) NSFNET with varying number of WCs

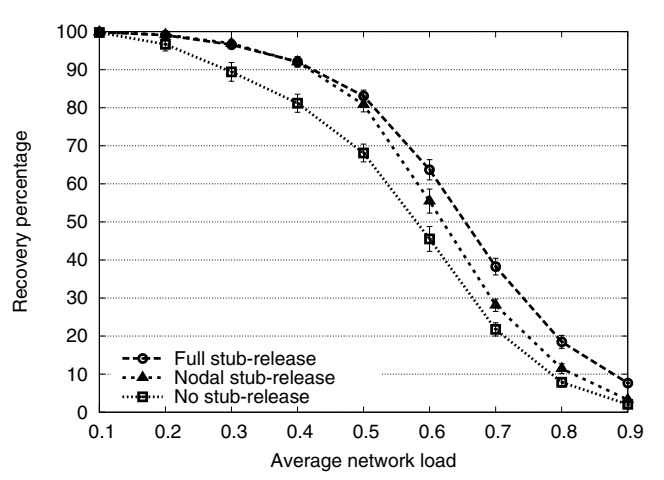

(d) NSFNET with varying network load

Fig. 2. Recovery percentages for evaluated network topologies with varying number of WCs (a)-(c) and varying network load (d).

can be used to compensate for the occupied resources in the no stub-release scheme.

In (b) NSFNET (i.e. the sparsest tested topology), if few WCs are available, the nodal stub-release method achieves a recovery percentage which matches the one of full stubrelease, while the no stub-release method has considerably lower performance. This is due to the fact that WCs are a limiting factor for successful recovery, and the nodal stubrelease scheme makes them available. When many WCs per node are provided, they are no longer the limiting factor, and only full stub-release, which can also release span resources, can get a slightly better performance.

In (c) Pan-European network, results are similar to (b), but the network gets span-limited at more WCs per node due to its larger size. The strength of the nodal stub-release scheme is especially dominant when WCs are sparse: nodal stub-release can significantly increase the restoration percentage compared to the case of no stub-release, while only a minor performance penalty compared to full stub-release is observable.

In (d) NSFNET with varying load, the recovery percentage is depicted as a function of the network load, keeping the number of WCs per node fixed to 5. At all loads, nodal and full stub-release achieve better recovery percentages than no stubrelease. At low loads, the nodal and full stub-release methods achieve the same performance, because span resources are widely available. As the network load increases, the performance of the nodal stub-release method approaches the no stub-release method's performance, as restoration success now mainly depends on the availability of span resources. (Similar results from other networks omitted due to space constraints.)

\section{CONCLUSION}

In this paper, we propose a nodal stub-release method, where only node resources (i.e. WCs) are released during stub-release actions. The scheme makes the scarce WC resources available in the recovery phase and allows fast and simple reversion to the pre-failure network configuration as re-negotiation of span resources is avoided. Simulation results show that the method performs well in both dense and sparse topologies and is especially useful when WCs are sparse in low-medium loaded networks. Under these conditions, the scheme matches the performance of full stub-release, and it always matches or outperforms the no stub-release scheme.

\section{REFERENCES}

[1] K.-C. Lee and V. O. K. Li, "A wavelength-convertible optical network," IEEE J. Lightwave Technol., vol. 11, no. 5, pp. 962-970, 1993.

[2] M. Ali, "Generalized sharing in survivable optical networks," IEEE/ACM Trans. Networking, vol. 14, no. 6, pp. 1388-1399, Dec. 2006.

[3] W.D. Grover, Mesh-Based Survivable Networks-Options and Strategies for Optical, MPLS, SONET, and ATM Networking. Prentice-Hall, 2004.

[4] M. Azim et al., "Models of restoration probability in WDM networks employing active restoration," Photonic Network Commun., vol. 10, no. 2, pp. 141-153, 2005.

[5] S. Ruepp, J. Buron, and N. Andriolli, "Increasing restorability for local-to-egress restoration in GMPLS controlled networks with limited wavelength conversion," in Proc. ICCS 2006, Oct. 2006.

[6] D. Schupke and R. Prinz, "Capacity efficiency and restorability of path protection and rerouting in WDM networks subject to dual failures," Photonic Network Commun., vol. 8, no. 2, pp. 191-207, 2004.

[7] L. Berger (ed.), "Generalized Multi-Protocol Label Switching (GMPLS) signaling functional description,” RFC 3471, Jan. 2003.

[8] OPNET Technologies, Inc., http://www.opnet.com. 